PROCEEDINGS OF THE

AMERICAN MATHEMATICAL SOCIETY

Volume 131, Number 7 , Pages 2155-2160

S 0002-9939(02)06890-9

Article electronically published on November 13, 2002

\title{
PERIODIC SOLUTIONS TO A DIFFERENCE EQUATION WITH MAXIMUM
}

\author{
H. D. VOULOV
}

(Communicated by Carmen C. Chicone)

\begin{abstract}
An open problem posed by G. Ladas is to investigate the difference equation

$$
x_{n}=\max \left\{\frac{A}{x_{n-1}}, \frac{B}{x_{n-3}}, \frac{C}{x_{n-5}}\right\}, \quad n=0,1, \ldots,
$$

where $A, B, C$ are any nonnegative real numbers with $A+B+C>0$. We prove that there exists a positive integer $T$ such that every positive solution of this equation is eventually periodic of period $T$.
\end{abstract}

\section{INTRODUCTION}

This note is motivated by the following open problem posed by G. Ladas 1 Problem 4.3.2].

Open Problem 1. Let $A, B, C$ be nonnegative real numbers and $A+B+C>0$. Investigate the properties of the positive solutions of the difference equations

$$
x_{n}=\max \left\{\frac{A}{x_{n-1}}, \frac{B}{x_{n-3}}, \frac{C}{x_{n-5}}\right\}, \quad n=0,1, \ldots,
$$

and

$$
y_{n}=\max \left\{A, B \frac{y_{n-1}}{y_{n-2}}, C \frac{y_{n-1} y_{n-3}}{y_{n-2} y_{n-4}}\right\}, \quad n=0,1, \ldots .
$$

Equation (1.1) is a special case of the equation

$$
x_{n}=\max \left\{\frac{A_{1}}{x_{n-1}}, \frac{A_{2}}{x_{n-2}}, \ldots, \frac{A_{p}}{x_{n-p}}\right\}, \quad n=0,1, \ldots,
$$

where the coefficients $A_{1}, A_{2}, \ldots, A_{p}$ are nonnegative real numbers, $A_{1}+A_{2}+\cdots+$ $A_{p}>0$, and the initial values $x_{-1}, x_{-2}, \ldots, x_{-p}$ are any given positive numbers. We call (1.3) the reciprocal difference equation with maximum, since it is a natural generalization of the simple reciprocal equation

$$
x_{n}=\frac{A_{q}}{x_{n-q}}
$$

which we obtain from (1.3) when $A_{q}$ is the only nonzero coefficient. Observe that each term of the sequence $x_{n}$ defined by (1.3) satisfies (1.4) for some $q$. However, $q$ depends on the previous terms $x_{n-1}, \ldots, x_{n-p}$. So (1.3) works as a switch, similar

Received by the editors February 20, 2002.

2000 Mathematics Subject Classification. Primary 39A10.

Key words and phrases. Periodic solutions, nonlinear difference equations. 
to the famous Collatz $(3 x+1)$ problem [2. Based on computer experiments, the positive solutions of (1.3) seemed to be eventually periodic of some period $T$. That is, $x_{n+T}=x_{n}$ for every $n \geq n_{0}$, where $T$ and $n_{0}$ may depend on the coefficients $A_{1}, \ldots, A_{p}$ and the initial conditions $x_{-1}, x_{-2}, \ldots, x_{-p}$. This lead to the following conjecture of G. Ladas [3. Conjecture 2.3.1].

Conjecture 1. Every positive solution of (1.3) is eventually periodic. Moreover, if $A_{q}>\max \left\{A_{j}: j \neq q, j \in\{1,2, \ldots, p\}\right\}$, that is, when the coefficient $A_{q}$ is dominating, then every positive solution of (1.3) is eventually periodic with period $T=2 q$.

In the case $p=2$, this conjecture was confirmed in [4]. When $p=3$, this conjecture was confirmed in [5], in the particular case when $A_{2}=0$. More precisely, it has been proved that every positive solution of the equation

$$
x_{n}=\max \left\{\frac{A_{1}}{x_{n-1}}, \frac{A_{3}}{x_{n-3}}\right\}
$$

is eventually periodic of period $T$, where $T=2$ provided $A_{1} \geq A_{3}$ and $T=6$ provided $A_{1}<A_{3}$. Observe that this result also solves Open Problem 1 in the special case when $C=0$.

Recently, Conjecture 1] was confirmed in [6] in the more general case when $p$ is arbitrary and when (1.3) has at most two nonzero coefficients. Clearly, this result solves Open Problem 1 in the special case when $A B C=0$.

In this paper we solve Open Problem 1 in the remaining case $A B C>0$. We also confirm Conjecture 1 in the special case when (1.3) reduces to (1.1). To the best of our knowledge, this is the first nontrivial result about (1.3) when there are more than two positive coefficients.

Our method of proof employs the fact that (1.1) and (1.2) are dynamically equivalent (by the substitution $y_{n}=x_{n} x_{n-1}$ ) and reduces the case $A B C>0$ to the case $A B C=0$, which has been solved in [6]. We believe that the results which we present here might yield insight into the study of the general equation (1.3) and possibly lead to a proof of Conjecture 1 (by induction) in the general case which remains open.

\section{Main Result AND SOME Discussions}

Recall that a sequence $\left\{x_{n}\right\}$ is said to be eventually periodic of period $T$ if an only if it eventually becomes periodic of period $T$, that is, there exists an integer $n_{0}$ such that

$$
x_{n+T}=x_{n} \text { for every } n \geq n_{0} .
$$

The minimal number $t$ which may be a period of any eventually periodic sequence $x_{n}$ is called the prime period of $\left\{x_{n}\right\}$.

Let $A, B, C$ be any nonnegative real numbers, $A+B+C>0$ and define the integer $T$ as follows:

$$
T= \begin{cases}2 & \text { when } A=B \geq C \text { or } A>\max \{B, C\}, \\ 6 & \text { when } A=C>B \text { or } B>\max \{A, C\}, \\ 8 & \text { when } B=C>A, \\ 10 & \text { when } C>\max \{A, B\} .\end{cases}
$$


Our main result is the following theorem.

Theorem 2.1. Let $T$ be defined by (2.1). Then the following statements are true:

(i) There exists a prime period $T$ solution of (1.1).

(ii) Every positive solution of (1.1) is eventually periodic of (not necessarily prime) period $T$.

Clearly, for some initial values, the solution $x_{n}$ of (1.1) may be eventually periodic of a prime period $t$, such that $t<T$ and $t$ is a factor of $T$. A trivial example is the equilibrium

$$
x_{n}=\bar{x}=\max \{\sqrt{A}, \sqrt{B}, \sqrt{C}\}
$$

which is periodic of prime period $t=1$, and, hence, it is periodic of any period $T \geq 1$.

As we indicated in the Introduction, in the special case when $A B C=0$, the proof of Theorem 2.1 follows from the results recently established in [6]. These results concern the following difference equation:

$$
x_{n}=\max \left\{\frac{P}{x_{n-k}}, \frac{Q}{x_{n-m}}\right\}, \quad n=0,1, \ldots,
$$

and they are summarized in the following theorem.

Theorem $2.2([6])$. Let $k, m$ be any positive integers with $k<m$. Let $P, Q$ be any positive real numbers and let $T$ be defined as follows:

$$
T= \begin{cases}2 k & \text { when } P>Q \text { or } P=Q, m=3 k, \\ 2 m & \text { when } P<Q, \\ m+k & \text { when } P=Q, m \neq 3 k\end{cases}
$$

Then the following statements are true:

(i) There exists prime period $T$ solutions of (2.2).

(ii) Every positive solution of (2.2) is eventually periodic of (not necessarily prime) period $T$.

The proof of Theorem 2.1 in the remaining case $A B C>0$, will be presented in the next section.

The following substitution

$$
x_{n} x_{n-1}=y_{n}
$$

suggested by G. Ladas, transforms (1.1) into (1.2). This observation is essential for our proof, and for the reader's convenience, we give its precise statement in the following lemma.

Lemma 2.1. The following statements are true:

(i) If $\left\{x_{n}\right\}_{n=-5}^{\infty}$ is a positive solution of (1.1), then the sequence $\left\{y_{n}\right\}_{n=-4}^{\infty}$ defined by (2.3) is a positive solution of (1.2).

(ii) If $\left\{y_{n}\right\}_{n=-4}^{\infty}$ is a positive solution of (1.2), then, for every $a>0$, the initial values

$$
x_{-1}=a, \quad x_{k-1}=\frac{y_{k}}{x_{k}}, \quad k=-1, \ldots,-4,
$$

generate a positive solution, $\left\{x_{n}\right\}_{n=-5}^{\infty}$, of (1.1) such that (2.3) holds for every $n \geq-4$. 
The proof follows immediately by (1.1), (1.2), and (2.3), and it will be omitted.

By Lemma 2.1, we see that Theorem 2.1 solves Open Problem 1 not only for (1.1) but for (1.2) as well.

Before we proceed with the proof of Theorem 2.1 it will be convenient to show that we may assume

$$
\max \{A, B, C\}=1
$$

without loss of generality. This follows immediately by the substitution $x_{n}=z_{n} \sqrt{\mathcal{D}}$, where $D=\max \{A, B, C\}>0$.

\section{Proof of Theorem 2.1}

In this section we will assume without loss of generality that $\max \{A, B, C\}=1$. We will also need the following lemma.

Lemma 3.1. Let $\max \{A, B, C\}=1$. Let $\left\{y_{n}\right\}_{n=-4}^{\infty}$ be any positive solution of (1.2). Then for every $n \geq 0$ be following inequalities hold:

$$
\begin{aligned}
A & \leq y_{n}, \\
B y_{n-1} & \leq y_{n} y_{n-2}, \\
C y_{n-1} y_{n-3} & \leq y_{n} y_{n-2} y_{n-4} .
\end{aligned}
$$

Additionally, if $A B>0$, then

$$
\frac{y_{n}}{y_{n-1}} \leq \max \left\{1, \frac{B}{A}, \frac{C}{B}\right\} \quad \text { for every } n \geq 2
$$

and

$$
y_{n} \leq \max \left\{\frac{1}{A}, \frac{C A}{B}\right\} \quad \text { for every } n \geq 5 .
$$

Proof. The inequalities (3.1), (3.2), and (3.3) follow immediately from (1.2), since $y_{n}>0$ for all $n \geq-4$. Then, for every $n \geq 2$, (1.2) implies

$$
\frac{y_{n}}{y_{n-1}}=\max \left\{\frac{A}{y_{n-1}}, \frac{B}{y_{n-2}}, \frac{C y_{n-3}}{y_{n-2} y_{n-4}}\right\}
$$

and (3.4) follows by (3.1) and (3.2). For every $n \geq 4$, (1.2) and inequalities (3.1) and (3.2) yield

$$
\frac{y_{n} y_{n-2}}{y_{n-1} y_{n-3}}=\max \left\{\frac{A y_{n-2}}{y_{n-1} y_{n-3}}, \frac{B}{y_{n-3}}, \frac{C}{y_{n-4}}\right\} \leq \max \left\{\frac{A}{B}, \frac{B}{A}, \frac{C}{A}\right\} .
$$

Therefore, for every $n \geq 5$, by (1.2), (3.4), and (3.6) we obtain

$$
y_{n} \leq \max \left\{A, B, \frac{B^{2}}{A}, C, \frac{C A}{B}, \frac{C B}{A}, \frac{C^{2}}{A}\right\},
$$

which implies (3.5) since $\max \{A, B, C\}=1$. The proof is complete.

Proof of Theorem 2.1. With no loss of generality we assume that $\max \{A, B, C\}=$ 1.

(i) When $T=2$, the result follows from the fact that the sequence $\left\{x_{n}\right\}$, defined by $x_{2 n}=a, x_{2 n-1}=\frac{1}{a}$ for $n \geq-2, a>0$, is a prime period two solution of (1.1). 
When $T=8$, we have $B=C=1>A$, and it is easy to see that the sequence $\left\{x_{n}\right\}$, defined by $x_{8 s}=A, x_{8 s-3}=x_{8 s-5}=\frac{1}{A}$ for every $s \geq 0$ and $x_{n}=1$ otherwise, is a prime period 8 solution of (1.1).

Let $T=10$. Then $C=1>\max \{A, B\}=F$ and similarly the sequence $\left\{x_{n}\right\}$ defined by $x_{10 s}=F, x_{10 s-5}=\frac{1}{F}$ for every $s \geq 0$ and $x_{n}=1$ otherwise, is a prime period 10 solution of (1.1).

Let $T=6$. Then two different classes are possible. When $B=1>\max \{A, C\}=$ $F$, we define $\left\{x_{n}\right\}$ by $x_{6 s}=F, x_{6 s-3}=\frac{1}{F}$ for $s \geq 0$ and $x_{n}=1$ otherwise. When $A=C=1>B$, then we define $\left\{x_{n}\right\}$ by $x_{6 s}=B, x_{6 s-1}=x_{6 s-5}=\frac{1}{B}$ for every $s \geq 0$ and $x_{n}=1$ otherwise. In both cases, $\left\{x_{n}\right\}$ is a prime period 6 solution of (1.1).

(ii) When $A B C=0$ the proof follows by Theorem 2.2. So we may assume that $A B C>0$ and $\max \{A, B, C\}=1$. Let $\left\{x_{n}\right\}_{n=-5}^{\infty}$ be a positive solution of (1.1). Lemma 2.1 implies that the sequence $\left\{y_{n}\right\}_{n=-4}^{\infty}$, defined by $y_{n}=x_{n} x_{n-1}$, satisfies (1.2). Let $T$ be the positive integer defined by (2.1). In each of the following five exhaustive cases, we will prove that $x_{n}$ is eventually periodic of period $T$.

Case $A=B=C=1$. In this case, by Lemma 3.1 we have $x_{n} x_{n-1}=y_{n}=1$ for every $n \geq 5$ and the result follows, since $T=2$.

Case $A=1>B C$. In this case, by Lemma 3.1 we obtain

$$
B \frac{y_{n}}{y_{n-1}} \leq \max \{B, C\} \leq 1=A \text { for every } n \geq 2 .
$$

Therefore, for every $n \geq 3$, (1.2) implies

$$
y_{n}=\max \left\{1, C \frac{y_{n-1} y_{n-3}}{y_{n-2} y_{n-4}}\right\}
$$

which yields

$$
x_{n}=\max \left\{\frac{1}{x_{n-1}}, \frac{C}{x_{n-5}}\right\} \quad \text { for } n \geq 3 .
$$

Then, by Theorem $2.2 x_{n}$ is eventually periodic of period $T$, where $T=2$ provided $C<1$ and $T=6$ provided $C=1$.

Case $B=1>A$. In this case, Lemma 3.1 implies

$$
\frac{A y_{n-2}}{y_{n-1}} \leq A y_{n-3} \leq \max \left\{1, C A^{2}\right\}=1
$$

for every $n \geq 8$, and by (1.2) we obtain

$$
y_{n}=\max \left\{\frac{y_{n-1}}{y_{n-2}}, \frac{C y_{n-1} y_{n-3}}{y_{n-2} y_{n-4}}\right\} .
$$

Therefore, for every $n \geq 8$, we have

$$
x_{n}=\max \left\{\frac{1}{y_{n-3}}, \frac{C}{y_{n-5}}\right\}
$$

and the result follows by Theorem 2.2 with $T=6$ for $C<1$ and $T=8$ for $C=1$.

Case $C=1>\max \{A, B\}$. In this case $T=10$. When $B \geq A^{2}$, Lemma 3.1 implies $A y_{n} \leq 1$ for every $n \geq 5$ and, moreover,

$$
\frac{y_{n-1} y_{n-3}}{y_{n-2} y_{n-4}} \geq \frac{1}{y_{n-5}} \geq A \quad \text { for } n \geq 10 \text {. }
$$


Then by (1.2) we obtain

$$
y_{n}=\max \left\{B \frac{y_{n-1}}{y_{n-2}}, \frac{y_{n-1} y_{n-3}}{y_{n-2} y_{n-4}}\right\}
$$

and

$$
x_{n}=\max \left\{\frac{B}{x_{n-3}}, \frac{1}{x_{n-5}}\right\} .
$$

Hence the result follows by Theorem 2.2

When $B<A^{2}$, for every $n \geq 9$, Lemma 3.1 implies

$$
y_{n-4} \leq \max \left\{\frac{1}{A}, \frac{A}{B}\right\}=\frac{A}{B} \quad \text { and } \quad y_{n-3} \geq A
$$

which yields

$$
\frac{y_{n-1} y_{n-3}}{y_{n-2} y_{n-4}} \geq \frac{y_{n-1}}{y_{n-2}} B .
$$

Then, by (1.2) we obtain

$$
y_{n}=\max \left\{A, \frac{y_{n-1} y_{n-3}}{y_{n-2} y_{n-4}}\right\}
$$

and

$$
x_{n}=\max \left\{\frac{A}{x_{n-1}}, \frac{1}{x_{n-5}}\right\} \text { for every } n \geq 9 .
$$

Hence, by Theorem 2.2 the proof is complete.

\section{REFERENCES}

[1] G. Ladas, Open problems and conjectures, J. Diff. Eqns. and Appl., 4(3)(1998), 312.

[2] D. Clark and J.T. Lewis, A Collatz-type difference equation, Congressus Numeratium, 111(1995), 129-135. MR 98b:11008

[3] G. Ladas, Open problems and conjectures, J. Diff. Eqns. and Appl., 2(1996), 339-341.

[4] A.M. Amleh, J. Hoag, and G. Ladas, A difference equation with eventually periodic solutions, Computers and Mathematics with Applications, 36(1998), 401-404. MR 99j:39002

[5] D. Mishev, W.T. Patula, and H.D. Voulov, On a Reciprocal Difference Equation with Maximum, Computers and Mathematics with Applications, 43(2002), 1021-1026.

[6] H.D. Voulov, On the Periodic Character of Some Difference Equations, J. Diff. Eqns. and Appl., 8(9)(2002), 799-810.

Department of Mathematics, Southern Illinois University at Carbondale, CarbonDALE, ILLINOIS 62901-4408

E-mail address: voulovh@yahoo.com 